\title{
Photo-graphies
}

\section{Entretien avec Vincent Capt - Droit au brut !}

\section{Rudolf Mahrer}

\section{OpenEdition}

\section{Journals}

Édition électronique

URL : http://journals.openedition.org/genesis/1496

DOI : 10.4000/genesis.1496

ISSN : 2268-1590

\section{Éditeur :}

Presses universitaires de Paris Sorbonne (PUPS), Société internationale de génétique artistique littéraire et scientifique (SIGALES)

\section{Édition imprimée}

Date de publication : 15 avril 2015

Pagination : 187-193

ISBN : 978-2-84050-992-9

ISSN : $1167-5101$

\section{Référence électronique}

Rudolf Mahrer, « Entretien avec Vincent Capt - Droit au brut ! », Genesis [En ligne], 40 | 2015, mis en ligne le 04 avril 2017, consulté le 16 mai 2019. URL : http://journals.openedition.org/genesis/1496 DOI : 10.4000/genesis. 1496 
Vincent Capt

Droit au brut !

\author{
Entretien avec Rudolf Mahrer
}

Pour la deuxième fois, Genesis accueille une étude consacrée à l'art brut : transcription et commentaire par Fanny Rojat d'une lettre d'Henri Bessaud-Narboux (ci-dessus)1. Voilà qui nous a paru une belle occasion d'interroger la rencontre entre critique génétique et poétique des écrits bruts - ce d'autant plus que l'intérêt pour la part verbale des auvres brutes va grandissant.

Si elle a lieu dans nos pages, une telle rencontre a-t-elle lieu concrètement, c'est-à-dire en théorie et en méthode dans la recherche sur les écrits bruts? Et si le dialogue reste à établir : est-il souhaitable, ou même pensable ? Voilà ce que nous avons voulu apprendre de Vincent Capt et de son livre Poétique des écrits bruts, paru en 2013 chez LambertLucas, en coédition avec la Collection de l'Art Brut de Lausanne 2.

Rudolf Mahrer - Cher Vincent, tes recherches t'ont conduit à Lausanne dans les archives de la Collection de l'Art Brut (CAB) : tu y as notamment transcrit, reproduit, commenté des documents écrits qui n'étaient pas voués à la publication. Dit comme ça, cela ressemble à un travail de généticien! Pourtant, si tu convoques plusieurs sciences du langage, dont la philologie, l'analyse du discours ou la psychanalyse, tu n'invites pas la critique génétique. Pourquoi?

Vincent Capt - Un pan de mon travail a en effet consisté à proposer des états de texte susceptibles de faire l'objet d'une analyse linguistique. La première étape dans cette direction consiste à établir des transcriptions. L'opération peut être délicate : tu t'en convaincras en regardant un feuillet de Justine Python (fig. 1). Dans Poétique des écrits bruts, les transcriptions sont ainsi regroupées en fin de travail, en regard des manuscrits donc, et constituent une partie du corpus global de réflexion.
Mais au-delà de ces similitudes de matériau et en partie de méthode, si je n'ai pas mobilisé la critique génétique, c'est que pour ma part, il ne s'agissait nullement de rendre compte de la dimension processuelle de l'écriture. Très modestement, j'avais pour but de rendre lisible un ensemble de textes, sinon uniquement visible.

Pour ce qui concerne les textes de Justine Python, mes transcriptions comportent un système de numérotation des lignes qui aide le lecteur à suivre l'analyse. Pour

1. Genesis a publié en 1995 un article de Michel Thévoz intitulé «L'écriture incestueuse » $\left(\mathrm{n}^{\circ} 7, \mathrm{p} .49-67\right)$.

2. Vincent Capt a soutenu en mai 2012 une thèse qui est à l'origine de Poétique des écrits bruts. Il a publié également, en 2012, Écrivainer. La langue morcelée de Samuel Daiber (Lausanne, Collection de l'Art Brut et Infolio) et, tout récemment, un recueil d'études autour des écrits bruts, Affoler la langue (Archipel, $\mathrm{n}^{\circ} 37$, décembre 2014), coédité par la Collection de l'Art Brut. Comme Fanny Rojat, enfin, il est cofondateur du Collectif de réflexion autour de 1'art brut (CrAB, <http://collectifartbrut.blogspot.com>). 


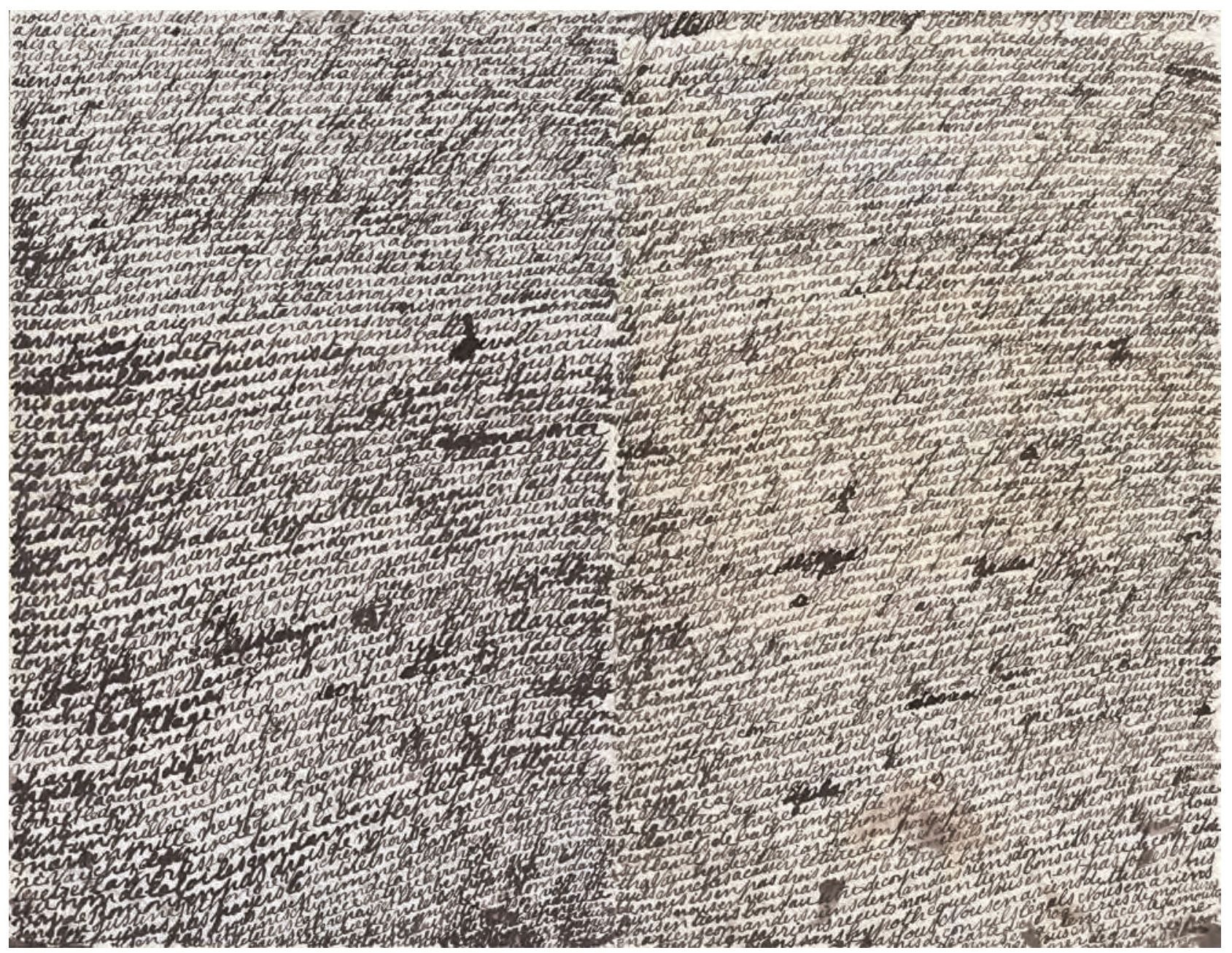

Fig. 1 : Justine Python, sans titre, 1932, encre sur papier, $21 \times 27 \mathrm{~cm}$.

On trouve une transcription de ce feuillet dans Poétique des écrits bruts, op. cit., p. 343 sq.

(C) Collection de l'Art Brut, Lausanne

ce stade initial de ma recherche, je n'ai pas interrogé le statut d'archive, de document, de pièce voire d'œuvre attribué aux textes ; j'ai fait jouer une sorte d'argument « pragmatique »- le confort de lecture, disons -, tout en étant conscient que la transcription est elle-même déjà un geste de sémantisation, un discours, en l'occurrence un acte éditorial effectué sur des manuscrits.

J'ajouterais qu'il m'a semblé important dans Poétique des écrits bruts de donner à lire ces archives dans leur intégralité, car leur accessibilité est généralement restreinte et fragmentaire ; on n'y accède souvent que dans le cadre d'expositions et dans certains ouvrages à titre d'illustration.
En effet, cent vingt pages de ton livre présentent des reproductions et des transcriptions. Était-ce la première fois que ces lettres, par exemple celles de Justine Python, subissaient cette réduction "de l'image au langage »?

Non, pour le corpus Python, un collaborateur du musée lausannois, Vincent Monod, avait réalisé une première transcription. J'ai confronté ses « relevés » aux manuscrits et je les ai modifiés, si nécessaire. En fait, j'ai surtout été amené à uniformiser la transcription et à respecter la plupart des spécificités observables, comme les rares espaces entre les unités graphiques ainsi que les alinéas ponctuant des lignes d'empans très variables. 
Plus généralement, pour les écrits bruts, ce geste de transcription a une histoire. Un recueil de textes asilaires, désormais fameux dans le milieu de l'art brut, a pour ainsi dire fondé la catégorie - même si Dubuffet avait déjà transcrit certains des écrits qu'il avait découverts. Intitulé Écrits bruts, coordonné en 1979 par l'ancien conservateur de la CAB, Michel Thévoz, avec la collaboration de Geneviève Roulin, l'ouvrage est exclusivement composé de transcriptions. Assez nombreux ont été ensuite les catalogues ou les monographies à proposer quelques transcriptions de textes bruts, très rarement avec un appareil critique cependant explicitant les choix et les perspectives de l'édition. N'ayant pas pour but de rendre compte de tous les détails du manuscrit, bien des transcriptions sont imprécises. C'est d'ailleurs ce « manque de rigueur » qu'interprète Fanny Rojat ici même à propos du corpus d'Henri Bessaud.

Tu expliques dans ton livre qu'il n'y a pas de brouillons parmi les écrits bruts conservés à la CAB, pas de textes préparant d'autres textes conservés eux aussi.

C'est exact, a priori. Initialement, la grande majorité des écrits bruts correspondent à des actes de communication accomplie au service d'une visée pragmatique explicite : principalement demander la libération d'un asile. Ce qui a été transmis notamment aux aliénistes avait donc valeur, matériellement, d'objet terminé.

En revanche, certaines œuvres apparentées aux écrits bruts sont des plans ou des schémas expliquant et préparant des objets tridimensionnels, comme des machines mécaniques ou technologiques. Le cas de l'œuvre de Jean Perdrizet, dont une partie est conservée aux Archives nationales à Pierrefitte, comprend clairement de telles formes préparatoires. Seuls les plans ont été gardés alors que les objets pourtant réalisés ont été détruits. C'est la figure du savant fou héritée surtout du XIX $X^{\mathrm{e}}$ siècle qui a été activée pour justifier l'intégration de ces plans au domaine de l'art. Jean Perdrizet proposait notamment des plans tirés en héliogramme sur du papier de tirage. Il pouvait ainsi les reproduire à son bon vouloir, et les recouvrir ensuite de couleurs et de textes chaque fois nouveaux.
Penses-tu que cette absence de brouillon soit un accident de conservation (tu expliques d'ailleurs que très peu de documents ont été conservés) ou penses-tu que ce soit définitoire de l'écrit brut? Il n'a pas de genèse, il ne se prépare pas.

Pour te répondre il faut notamment tenir compte des circonstances de la première conservation des textes asilaires, dans les archives médicales. Rarement retenus, ils avaient valeur d'échantillon représentatif de symptômes psychiatriques. De ce fait, il n'est guère étonnant qu'aucun écrit préparatoire n'ait été conservé. Mais cela n'implique pas pour autant qu'aucun brouillon n'ait été réalisé... Quand on voit notamment le soin apporté à certaines réalisations, l'équilibre dispositionnel de certaines productions, il ne serait pas surprenant d'apprendre que les futurs auteurs d'écrits bruts faisaient des plans ou au moins des essais. Jusque-là, nous ne disposons simplement pas dans les archives de preuves attestant de la préparation d'un écrit à venir.

Tu disais tout à l'heure que la plupart des écrits bruts s'interprètent comme "des actes de communication accomplie». Est-ce à dire qu'ils ne présentent pas de ratures?

Non, pas nécessairement. Mais elles sont plutôt rares. À mon sens, on peut donner à cette relative rareté au moins deux raisons. L'une psychologisante et l'autre ayant peut-être quelque rapport avec la génétique. Les ratures observables, par exemple dans les textes de Samuel Daiber, ou bien les marques ostensibles d'ajout rédigées tête-bêche et entre deux lignes chez Justine Python, sont l'indication d'une reprise et d'un retour sur la production écrite. Ces réalisations a posteriori sont le signe d'une certaine réflexivité. Il est cependant loisible d'interpréter leur rareté relativement à l'état psychique du scripteur, potentiellement «plongé dans son délire », relisant peu et négociant peu son propre acte d'écriture. L'autre hypothèse consiste à considérer la faible présence des ratures comme la résultante d'écritures orientées préalablement non par des brouillons préparatoires, mais par des réalisations préexistantes, nombreuses et similaires notamment sur le plan graphique et compositionnel. 
L'absence de brouillons s'expliquerait donc également par la forte uniformité des productions asilaires : par exemple, les lettres de Gaspard Corpataux que tu m'as souvent montrées sont très homogènes, sur le plan graphique notamment. Les écrits bruts résulteraient d'écritures fortement ritualisées...

L'identité au moins graphique de certains textes est parfois si forte chez un même auteur qu'il est pertinent de penser qu'un même texte, dans son aspect schématique, a pu lui servir de «modèle » pour toute sa production. On aurait affaire là à un cas intéressant, où l'écrit n'a pas valeur stricto sensu d'avant-texte, il n'est pas un « pro-jet »... mais pour autant il est un moyen pour une production tierce. On aurait affaire à une démarche réalisée à partir d'une matrice spécifique, reconduite de façon constante texte après texte.

Cette piste semble intéressante. Elle indique que l'abandon de formes socialisées du discours pourrait provenir d'une subjectivation maximalisée et pour ainsi dire réduite par le scripteur à un seul mode d'énonciation écrite. L'identité forte des textes d'un même auteur expliquerait d'une part la rareté d'accidents génétiques et d'autre part l'attribution d'un jugement artistique. Ce processus de quasi « reproduction » d'une œuvre par l'autre favorise la reconnaissance d'une manière, à savoir un sujet identifié dans et par son écriture, et confondu avec elle.

De Dubuffet, on hérite une conception de l'art brut comme production instinctive, naturelle, spontanée; on voit dans le fou un sujet préservé de la rationalité inhérente à la socialisation : comme l'enfant, le primitif ou "le peuple ». La parole brute, non falsifiée par la culture littéraire-élitaire, ne doit pas l'être non plus par le travail patient et réfléchi de réécritures. L'art brut doit être spontané, impétueux et donc sans genèse. On sait aujourd' hui que les écrits dits "automatiques » présentent des ratures. Ne faut-il pas penser aussi que la spontanéité de l'écrit brut est un effet de lecture plutôt qu'une réalité de l'écriture?

L'art brut a en effet été fantasmé tel. Le spontanéisme, dont relève la conception du brut chez
Dubuffet et d'autres à sa suite, a contribué à faire valoir plus globalement un système de pensée primitiviste. Ce dernier résulte certainement de représentations de la folie provenant de la rhétorique médicale ellemême (via le paradigme de l'hystérie par exemple) et aussi de la photographie (la pratique du portrait d'aliénés). Cet imaginaire trouve en fait ces racines dans l'Antiquité... Aujourd'hui, certains acteurs du monde de l'art brut sont imprégnés de ce système de représentations. Je ne sais pas si leur positionnement est rétrograde ou anachronique, mais il a en tout cas pour effet de naturaliser des points de vue sur le langage et sur les œuvres.

Je tente de résister à cette forme de paresse intellectuelle, comme Dubuffet lui-même, qui était particulièrement sensible à la diversité des regards posés sur le monde. En inventant l'art brut, il a précisément proposé un point de vue nouveau sur certaines productions. Sa démarche est caractéristique d'un relativisme certain, confrontant diverses assignations. Actuellement, son perspectivisme semble mis de côté au profit d'une forme d'essentialisation de sa position.

Je vois un autre point de tension entre poétique de l'art brut et génétique : il réside dans la divergence profonde que tu décris entre le geste de lecture et le geste d'écriture. Le sujet-interprète voit de l'art là où le sujet-scripteur voyait de la communication, parfois de la contestation à visée politique, souvent une question de survie personnelle. Dès lors, ce que tu présentes fort bien, c'est le trajet générique qu'ont connu ces lettres : requêtes laissées sans réponse, elles sont devenues ensuite symptômes pour des médecins, classés sous secret médical, et enfin objets d'art dans des archives muséales consacrant des sortes d'auteurs.

Des « sortes d'auteurs », tu fais bien de modaliser. L'art brut, avant d'être une catégorie de l'histoire de l'art relative à certaines collections, provient d'une pensée de l'art et de son action critique sur la société. Les œuvres apparentées à l'art brut sont nomades et ont la vertu de «faire problème » à toute assignation. C'est là que réside leur véritable spécificité (et non dans leur forme ou une quelconque psyché préalable). Leur 
efficience consiste à interroger l'exclusivité de chacune des assignations dont elles ont fait l'objet. Par effet retour, c'est l'histoire collective d'une sensibilité au langage qui se trouve éclairée. Donc principalement une question de réception, oui. Une question qui unit théorie du langage et imaginaires de la langue.

Ce déplacement - de la production vers la réception - me paraît d'ailleurs renforcé par le fait que tu n'aies retenu dans ton corpus que des lettres? As-tu choisi le corpus à cette fin?

Le genre épistolaire est simplement majoritaire dans tous les écrits de l'enfermement : on écrit à une personne dont on est séparé physiquement et avec laquelle la communication est nécessairement décalée, reportée temporellement aussi. Plusieurs études confirment cette proéminence générique. Du point de vue d'une poétique, la logique de l'énonciation se trouve radicalisée et le poids de l'interprétant maximalisé : est prise alors en considération l'activité exercée par celui qui réagit verbalement. Le déséquilibre entre production et reconnaissance rappelle une formule de Jürgen Trabant, « le mot ne fait que disparaître en quelque sorte dans l'autre subjectivité 3 ». On pense à Volochinov aussi, chez qui toute énonciation enclenche de façon quasi réflexe la « compréhension responsive »d'autrui, qui n'écoute et interprète ce qu'il entend qu'en fonction de ses propres préoccupations (de son arrière-fond idéologique, de sa situation). En art, ce serait l'attitude du ready-made.

Ou justement la formule de Marcel Duchamp que tu cites également : "C'est le regardeur qui fait l'cuvre. " Mais quand même : tout écrit ne se laisse pas regarder comme brut? Ce que je veux dire, schématiquement, c'est que pour toi "les ouvres "brutes" n'existent qu'a posteriori ${ }^{4} »$, alors que l'objet de la critique génétique, c'est l'a priori de l'œuvre. Dès lors, cette dernière ne peut rien apporter à une poétique de l'écrit brut?

La génétique ne fait pas pour l'heure partie de la poétique globale des écrits bruts, centrée sur les modalités de lecture à inventer et la critique des attitudes conventionnelles de réception. Mais je serais le premier ravi de découvrir un dossier préparatoire parmi les écrits affiliés à l'art brut ! Il serait alors tout à fait envisageable que l'interprétant constitue un avant-texte et décrive les diverses « phases » de l'élaboration. Encore faut-il que ce type de dossier existe...

Par ailleurs, je pense que le point de vue de l'art sur une production présuppose (du moins présupposait aux yeux de Dubuffet) de retenir des «produits finis », à savoir des réalisations non préparatoires et ayant été transmises comme telles (parfois conservées pour soi), tout du moins des productions estimées abouties aux yeux de leur auteur. Les poétiques du processus sont un peu plus tardives dans l'histoire de l'art. Dans le continu, la génétique est elle aussi une forme historicisée du regard - scientifique - sur l'écriture. Dubuffet s'intéressait au déconditionnement final provoqué selon lui par certaines œuvres (comme effet d'un objet clos). Même s'il s'est beaucoup intéressé aux techniques et aux matériaux employés, ce n'est pas le processus de création pour lui-même qui a retenu son attention, sinon les tréfonds psychiques desquels celui-ci pouvait provenir.

Mais il y a bien dans ces " tréfonds », dans l'histoire de l'écriture et dans l'écrit lui-même, quelque chose qui prépare, qui attend ou qui fraie la lecture que la tradition interprétative "dubuffetienne » a institutionnalisée : celle de l'écrit asilaire comme œuvre d'art. Tu dis que "l'attribution du statut d'œuvre n'est pas relative à une propriété intrinsèque des textes, mais qu'elle correspond à un moment de leur historicité " (Poétique des écrits bruts, p. 74). N'est-ce pas trop fort? L'attitude de l'auditeur de Volochinov est "responsive ", c'est-à-dire qu'elle répond quand même à une sollicitation de l'énonciateur...

Oui, mais cette sollicitation n'est pas nécessairement adressée à l'auditeur. Dubuffet « répond » à des textes qui convoquent explicitement un autre destinataire que lui (souvent un médecin ou un membre de la famille).

3. Jürgen Trabant, Traditions de Humboldt, Paris, Éditions de la Maison des Sciences de l'homme, 1999, p. 38.

4. Poétique des écrits bruts, op. cit., p. 73-74, où Vincent Capt cite Gérard Dessons, La Manière folle. Essai sur la manie littéraire et artistique, Paris, Manucius, 2010, p. 8. 
Je pense que le contexte de production des écrits a fortement orienté les prospections de Dubuffet : l'asile (puis l'hôpital psychiatrique, dans une certaine mesure) était suffisamment isolé et coupé de la société pour favoriser l'émergence de productions défaites de conditionnement culturel ou du moins artistique. L'art brut se situe également dans le prolongement de certaines avant-gardes, dont le surréalisme, qui n'a eu de cesse d'explorer la folie voire de railler la psychiatrie. Choisir l'asile représentait une certaine logique de ce point de vue. Il faut ajouter à cela le fait que les patients internés travaillent à partir de matériaux pauvres, récupérés ici et là : cette esthétique de la modestie, de la parcimonie, plaisait à Dubuffet.

Enfin, bien souvent les demandes de libération sont cruellement répétées sur une longue période (parfois durant une vie d'enfermement) et il n'est pas impossible, comme nous l'esquissions plus haut, que l'invention d'une identité dans l'écriture se soit affirmée dans et par une pratique rédactionnelle répétée, parfois quotidienne, en tous les cas jamais longtemps interrompue.

Pour aborder le problème moins frontalement, Fanny Rojat ici même défend l'idée que l'édition des écrits de Henri Bessaud par Michel Thévoz et Geneviève Roulin (dans Écrits bruts) tendrait à obscurcir les textes et, en les conformant ainsi à une certaine conception de la littérature, à accentuer leur caractère créatif et littéraire? Partages-tu son analyse et penses-tu qu'elle soit valide au-delà du cas Bessaud?

Non, et de manière plus générale, c'est même selon moi le contraire. La tendance à la littérarisation des écrits d'aliénés a cependant été une pratique reconnue au début du $\mathrm{Xx}^{\mathrm{e}}$ siècle. La mise en circulation d'écrits bruts entreprise par Thévoz a d'abord permis de rendre simplement accessibles ces écrits sinon voués à l'anonymat. Il ne faut pas omettre le fait que la publication accompagnait à Lausanne une exposition homonyme cette année-là (1979). En outre, les transcriptions rendent lisibles des écrits sinon cryptiques pour la plupart. Dans le livre, Thévoz propose par ailleurs pour chaque corpus une brève introduction indiquant la provenance des textes et leur apparentement initial à un genre de discours. Si les textes transcrits changent de matérialité et de statut discursif, ils ne sont donc pas plus hermétiques pour autant, à mon avis.

Ce qui peut participer à littérariser les écrits bruts - à condition que l'on confine la littérature à un certain hermétisme, à un idéal en opposition au modèle de la belle langue -, c'est comme le dit Fanny Rojat de sélectionner les fragments spécialement obscurs au détriment d'autres plus accessibles, ou de présenter des transcriptions infidèles aux manuscrits qui étrangéifient plus encore certains énoncés.

Mais quand on lit l'introduction théorique que Michel Thévoz consacre en 1978 aux écrits bruts dans son ouvrage de référence Le Langage de la rupture, ce qui est surtout défendu, c'est une sociocritique des institutions (notamment littéraires) par l'intermédiaire des œuvres d'art brut. "La notion d'écrit brut se définira essentiellement par opposition à la littérature telle qu'on l'entend ordinairement » (p. 11). Thévoz insiste par ailleurs sur le fait que les auteurs d'écrits bruts n'entretiennent aucun rapport avec la sphère littéraire : " les auteurs d'écrits bruts [sont] étrangers aux milieux culturels et, à plus forte raison, à l'institution littéraire » (p. 69-70). Indexer les écrits bruts à la littérature, même comme marge, c'est ignorer leur criticité. Sur un fond de pensée antipsychiatrique, Thévoz a pour sa part travaillé de l'intérieur certaines catégories pour mieux les faire imploser aux souffles de productions irréductibles à toute forme d'indexation qui leur préexisterait.

Enfin, le recueil de ces écrits au sein d'un livre publié pourrait aussi les inscrire dans la sphère littéraire. Michel Thévoz participe d'une position paradoxale sur ce point, indiquant que "l'absence de toute perspective de publication intervient comme une caractéristique essentielle de ce que nous entendons par écrits bruts » (p. 131), alors même qu'il publie des extraits d'écrits bruts dans le même ouvrage (notamment). On peut nuancer ce paradoxe en précisant que les publications d'écrits bruts ne le sont jamais du fait de l'auteur, mais sont proposées comme objet de réflexion.

Notre discussion m'amène à penser que, ensemble, génétique et écrits bruts invitent à réfléchir à ce que certains psycholinguistes appellent la "productivité du 
déjà produit ». Les écrits bruts que j'ai lus en découvrant ton travail paraissent spectaculairement conduits par les mécanismes associatifs de la production verbale écrite (association sur le signifiant, sur le signifié, sur l'interdiscours...). Les paradigmes, que la génétique rencontre dans les ratures, constituent ici le tissu même de la textualité : ce ne sont plus des états différents de l'écrit qui témoignent d'un parcours génétique, mais la trame textuelle qui se déroule et se déploie comme un processus déterminé par ces mécanismes. On aurait envie de dire de l'écrit brut qu'il est lui-même, dans son déploiement spatial, la trace de son processus d'élaboration. Sa cohérence est sa progression : une dérive que libère peut-être le faible assujettissement de son sujet-scripteur au cadre générique et à la socialisation, et qui ouvre au dialogisme avec soi, c'est-à-dire avec sa langue et son interdiscours. Autrement dit, plus qu' un autre, le scripteur "brut » écrit en répondant à l'écoute qu'il accorde à sa propre parole (c'est le dialogisme intralocutif qui domine).
J'aime beaucoup l'expression « productivité du déjà écrit ». Michel Thévoz, dans l'ouvrage cité plus haut, avait indiqué ceci à propos du mode de production des écrits d'Aloïse Corbaz :

\begin{abstract}
Aloïse a commencé par copier le livret de la Fête des Vignerons à Vevey, qui nourrit tous les vingt-cinq ans les nostalgies terriennes de la population lémanique. Puis elle s'est mise peu à peu à interpoler, sans qu'on puisse délimiter précisément ce qui lui revient. Enfin elle s'est relue en annotant entre les lignes. Si elle avait eu la place, elle aurait encore annoté ses annotations. Cela nous éclaire sur le principe générateur de ses textes. [...] Il s'agit en quelque sorte d'un rewriting généralisé (Écrits bruts, Paris, PUF, 1979, p. 31).
\end{abstract}

Lisons attentivement cette citation et considérons-la comme une manière - interdiscursive (!) - de te répondre, par l'affirmative.

RUdolf Mahrer est maître-assistant en linguistique française à l'Université de Lausanne et responsable du séminaire « Manuscrit - Linguistique - Cognition » de l'ITEM (CNRS-ENS). Ses recherches portent sur l'énonciation écrite, notamment sous l'angle de ce qui apparente et différencie celle-ci de l'énonciation orale. 
